\title{
Assessment of Physical and Chemical hazards Among Health Care Workers in Wasit Governorate
}

\author{
Nassa Sattar Yaqoob Al-mayahi ${ }^{1}$, Shrouk Abdulrazk Hassan Alibraheem², Alaa Kadhim \\ Jasim $^{3}$ \\ \{nsystar09@ gmail.com ${ }^{1}$, shrouk.alibraheem@stu.edu.iq ${ }^{2}$, alaa-alderawi@ stu.edu.iq $\left.{ }^{3}\right\}$ \\ Southern Technical University, Health and Medical Technical College, Community Health Techniques \\ and Medical Laboratory Department//raq ${ }^{1,2,3}$
}

\begin{abstract}
Health care workers are on the front lines of this worldwide pandemic, with the significant burden of identifying and treating an increasingly expanding number of severely sick patients, sometimes making vital decisions under physical and psychological stress. This study is descriptive; a cross-sectional study conducted at four hospitals in Wasit governorate. The data collection started from December 2020 till March 2021. The Data were collected by direct interview with the health worker by the researcher, by using a selfreporting questionnaire from the occupation hazard dimension, which includes two domains: physical hazards and chemical hazards. The statistical method used includes Mean, Standard Deviation, and Chi-square. Statistical significance was considered whenever the P-value was equal to or less than 0.05 . The current study found that the highest percentage $254(57.6 \%)$ were in the age group <30 years, there was a distinct female's preponderance of $242(54.9 \%)$. There is a highly significant positive correlation ( $\mathrm{P}$-value $<0.01$ ) between physical hazards and prevention and control methods $\left(\mathrm{r}=0.144^{*}\right)$; a highly significant positive correlation $(\mathrm{P}$-value $<0.01)$ between chemical hazards, and prevention and control methods $\left(\mathrm{r}=0.770^{*}\right)$.where the chemical hazard reached to241(54.6\%) while followed physical hazards reached to 196(44.4\%) the results also showed Weighted Mean for physical hazard equal 3.28 \pm 0.67 which is within the medium level risk.
\end{abstract}

Keywords: Assessment, Physical, Chemical hazards, Health care workers.

\section{Introduction}

Occupational hazard in Iraq poses a danger to the individual in the working environment in addition to the consequences such as fatal accidents, minor to serious injuries and immediate allergic and systemic effects [1]. Occupational hazard is the risk, harm, or danger that an individual is exposed to at the workplace. Workers are exposed to a variety of risks throughout work times, virtually as many as the diverse types of labour, including physical, biological, mechanical, chemical, psychological, and ergonomic issues. These are to account for one of many negative health effects[2]. In the health sector at present, many health workers and other workers are exposed to the risks of ionizing and non-ionizing radiation that are used in daily use in medical tasks. It is considered of importance at the diagnostic and therapeutic levels [3]. Health workers, who are estimated to number more than two million, deal with radiation as a result of related practices, exposing half of this number to artificial and ionizing radiation [4]. The risks posed by chemicals are processed, produced, processed, and transported and they have an environmental and human health impact. A chemical hazard is a form of chemical exposure occupational hazard. Chemical occupational exposure can have short-term and long-term health 
effects. Neurotoxins, immunologic agents, dermatologic agents, carcinogens, toxic substances, reproductive substances, systemic toxins, asthma genes, and sensitizers, to mention just a few, are toxic substances [5]. In health-care environments such as hospitals, doctor's offices, and clinical labs, personal protective equipment (PPE) is done routinely. Personal protection equipment (PPE) acts as a barrier between infectious elements such as viruses and bacteria and the body, mouth, nose, and eyes (mucous membranes) when worn properly. PPE can stop contaminants from being spread by blood, bodily fluids, or respiratory secretions [6].

\subsection{Aims of the Study}

1. To assess physical and chemical hazards among health care workers in Wasit governorate.

2. To find out the relationship between physical and chemical hazards and health workers' demographic information and some related factors.

3. To find out the relationship between physical and chemical hazards and prevention and control methods.

\section{Methodology}

This study is a descriptive cross-sectional study conducted at four hospitals in Wasit governorate which are AL-zahraa teaching hospital, AL-suwaira hospitals, AL-naemania hospitals, and ALshaheed fairooz hospital. Data were collected during the period starting from December 2020 till March 2021. The worker's participation in the study were441 workers in these hospitals. Data were collected using direct interviews of health workers using a questionnaire. the questionnaire from consists of four-part: First part socio-demographic characteristic of the studied health worker regarding age, gender ...etc. The second part is the occupational characteristic of the studied health worker regarding professional, years of expertness, and place work. The third part the occupational hazard dimensions which include four domains: Physical hazard (14 items), Chemical hazard (11 items), and prevention and control methods (23 items) was assessed by using a five Likert scale. Analysis of data was carried out using the available statistical package of SPSS-25 (Statistical Packages for Social Sciences- version 25). Data were presented in simple measures of frequency, percentage, mean, standard deviation, and range (minimum-maximum values). The significance of difference for different percentages (qualitative data) was tested using the Pearson Chi-square test $(\chi 2$-test). Statistical significance was considered whenever the P-value was equal to or less than 0.05 .

\section{Results}

Table 1 represents the Socio-demographic characteristics of the study population. The results found that the mean $\pm \mathrm{SD}$ of their ages was $30.8 \pm 8.12$ years, the age range of the health worker in the study was between 20-56 years, The highest percentage 254(57.6\%) were in the age group $<30$ years. There was a distinct female preponderance 242 (54.9. As for residence and educational level, the study revealed that most participants in studied hospitals from urban regions $394(89.3 \%)$, while the highest percentage $354(80.3 \%)$ of health workers have an educational level (institute and College) followed by $74(16.8 \%)$ had secondary educational level the result of this study indicated that $265(60.1 \%$ ) of the study population were married and 
$161(36.5 \%)$ of participation were single in the study hospital. The study demonstrated that the first rank of health care workers were nurses 125 (28.3\%) followed by 107(24.3\%) of medical assistants, laboratory assistants, and radiology assistants in all studied hospitals. While the overall frequency of Technicians was $86(19.5 \%)$ in all hospitals. Which found that most participants had years' experience $\geq 5$ is $244(55.3 \%)$. The result of this study indicated that the highest proportion of participants were $117(26.5 \%)$ working in medical Laboratories followed by $82(18.6 \%)$ working in admission units for patients in all studied hospitals.

Table 1. Socio-demographic characteristics of health care workers.

\begin{tabular}{|c|c|c|c|}
\hline \multicolumn{2}{|c|}{ Socio-demographic characteristics } & \multirow{2}{*}{$\begin{array}{l}\text { No } \\
254\end{array}$} & \multirow{2}{*}{$\begin{array}{r}\text { Percent } \\
57.6\end{array}$} \\
\hline age groups & $<30$ years & & \\
\hline & $\geq 30$ years & 187 & 42.4 \\
\hline & Mean \pm SD(Range) & \multicolumn{2}{|c|}{$30.83 \pm 8.123(20-56)$} \\
\hline \multirow[t]{2}{*}{ Gender } & Male & 199 & 45.1 \\
\hline & Female & 242 & 54.9 \\
\hline \multirow[t]{2}{*}{ Residence } & Urban & 394 & 89.3 \\
\hline & Rural & 47 & 10.7 \\
\hline \multirow[t]{4}{*}{ Educational level } & Primary & 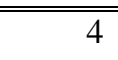 & 0.9 \\
\hline & Intermediate & 9 & 2.0 \\
\hline & Secondary & 74 & 16.8 \\
\hline & Institute and College & 354 & 80.3 \\
\hline \multirow[t]{3}{*}{ social status } & Married & 265 & 60.1 \\
\hline & Single & 161 & 36.5 \\
\hline & Others & 15 & 3.4 \\
\hline \multirow[t]{3}{*}{ experience years } & $<5$ years & 197 & 444.7 \\
\hline & $\geq 5$ years & 244 & 55.3 \\
\hline & Mean \pm SD(Range) & \multicolumn{2}{|c|}{$7.83 \pm 7222(1-35)$} \\
\hline \multirow[t]{3}{*}{ Place of working } & Administrative units & 22 & 5.0 \\
\hline & consulting unit & 10 & 2.3 \\
\hline & Radiology department & 16 & 3.6 \\
\hline
\end{tabular}




\begin{tabular}{|c|c|c|c|}
\hline & $\begin{array}{l}\text { Admission unit for } \\
\text { patients }\end{array}$ & 82 & 18.6 \\
\hline & Health units & 45 & 10.2 \\
\hline & Pharmacy unit & 44 & 10.0 \\
\hline & Emergency & 36 & 8.2 \\
\hline & Operation department & 45 & 10.2 \\
\hline & Blood bank & 9 & 2.0 \\
\hline & Medical Laboratories & 117 & 26.5 \\
\hline & Maternity hall & 15 & 3.4 \\
\hline \multirow[t]{7}{*}{ Professional } & Physicians & 29 & 6.6 \\
\hline & Pharmacists & 34 & 7.7 \\
\hline & Technicians & 86 & 19.5 \\
\hline & Nurse & 125 & 28.3 \\
\hline & Biologist and Chemist & 33 & 7.5 \\
\hline & $\begin{array}{l}\text { Medical assistant and } \\
\text { laboratory assistant and } \\
\text { Radiology }\end{array}$ & 107 & 24.3 \\
\hline & $\begin{array}{l}\text { Administrative } \\
\text { employees }\end{array}$ & 27 & 6.1 \\
\hline
\end{tabular}

Table 2 represent the distribution of HCW according to physical hazards in. showed that the mean of for question (1) ( conditioning is available in the workplace and suitable for use), was (3.57), for question (11) ( the electrical lifts are in good and safe condition ) was (2.59), for question (12) (enough lighting is available at the workplace )was (3.74). While the stander deviation for question (1)was (1.369), for question (11) was(1.323), was(1.176),for question(12) was (1.208).

Table 2. The Distribution of Healthcare Workers According to Physical Hazards.

\begin{tabular}{|c|c|c|c|c|c|c|c|c|c|c|c|c|}
\hline \multirow[t]{2}{*}{ Physical hazard } & \multicolumn{2}{|c|}{$\begin{array}{l}\text { Strongl } \\
\text { y agree }\end{array}$} & \multicolumn{2}{|c|}{ Agree } & \multicolumn{2}{|c|}{ Neutral } & \multicolumn{2}{|c|}{ Disagree } & \multicolumn{2}{|c|}{$\begin{array}{l}\text { Strongly } \\
\text { Disagree }\end{array}$} & $\begin{array}{l}\text { M } \\
\text { ea }\end{array}$ & \multirow[t]{2}{*}{$\mathrm{SD}$} \\
\hline & $\begin{array}{l}\mathrm{N} \\
\mathrm{O}\end{array}$ & $\%$ & No & $\%$ & No & $\%$ & No & $\%$ & No & $\%$ & $\mathrm{n}$ & \\
\hline
\end{tabular}




\begin{tabular}{|c|c|c|c|c|c|c|c|c|c|c|c|c|}
\hline $\begin{array}{l}\text { 1.Air conditioning } \\
\text { is available in the } \\
\text { workplace and } \\
\text { suitable for use }\end{array}$ & $\begin{array}{l}1 \\
2 \\
0\end{array}$ & $\begin{array}{l}2 \\
7 \\
2\end{array}$ & $\begin{array}{l}1 \\
8 \\
2\end{array}$ & $\begin{array}{r}41 \\
.3\end{array}$ & 33 & $\begin{array}{r}7 . \\
5\end{array}$ & $\begin{array}{l}4 \\
0\end{array}$ & $\begin{array}{r}9 . \\
1\end{array}$ & $\begin{array}{l}6 \\
6\end{array}$ & $\begin{array}{c}1 \\
5 . \\
0\end{array}$ & $\begin{array}{r}3 . \\
5 \\
7\end{array}$ & $\begin{array}{r}1.3 \\
69\end{array}$ \\
\hline $\begin{array}{l}\text { 2.Central } \\
\text { ventilation systems } \\
\text { are available in the } \\
\text { work environment }\end{array}$ & $\begin{array}{l}7 \\
6\end{array}$ & $\begin{array}{l}1 \\
7 \\
2 \\
\end{array}$ & $\begin{array}{l}1 \\
4 \\
3\end{array}$ & $\begin{array}{r}32 \\
.4\end{array}$ & 75 & $\begin{array}{c}1 \\
7 . \\
0\end{array}$ & $\begin{array}{l}7 \\
9\end{array}$ & $\begin{array}{c}1 \\
7 . \\
9\end{array}$ & $\begin{array}{l}6 \\
8\end{array}$ & $\begin{array}{c}1 \\
5 . \\
4\end{array}$ & $\begin{array}{c}3 . \\
1 \\
8\end{array}$ & $\begin{array}{r}1.3 \\
34\end{array}$ \\
\hline $\begin{array}{l}\text { 3.Windows is } \\
\text { available in the } \\
\text { work environment } \\
\text { and help to enter } \\
\text { lighting and air } \\
\text { circulation }\end{array}$ & $\begin{array}{l}1 \\
4 \\
6\end{array}$ & $\begin{array}{l}3 \\
3 \\
i\end{array}$ & $\begin{array}{l}1 \\
6 \\
3\end{array}$ & $\begin{array}{r}37 \\
.0\end{array}$ & 35 & $\begin{array}{r}7 . \\
9\end{array}$ & $\begin{array}{l}4 \\
2\end{array}$ & $\begin{array}{r}9 . \\
5\end{array}$ & $\begin{array}{l}5 \\
5\end{array}$ & $\begin{array}{r}1 \\
2 . \\
5\end{array}$ & $\begin{array}{c}3 . \\
6 \\
9\end{array}$ & $\begin{array}{r}1.3 \\
49\end{array}$ \\
\hline $\begin{array}{l}\text { 4.A sterilization } \\
\text { gate is available at } \\
\text { the entrances to the } \\
\text { health institution }\end{array}$ & $\begin{array}{l}1 \\
0 \\
0\end{array}$ & $\begin{array}{l}2 \\
2 \\
7 \\
\end{array}$ & $\begin{array}{l}1 \\
0 \\
7\end{array}$ & $\begin{array}{r}24 \\
.3\end{array}$ & 59 & $\begin{array}{c}1 \\
3 . \\
4\end{array}$ & $\begin{array}{l}9 \\
4\end{array}$ & $\begin{array}{c}2 \\
1 . \\
3\end{array}$ & $\begin{array}{l}8 \\
1\end{array}$ & $\begin{array}{c}1 \\
8 . \\
4\end{array}$ & $\begin{array}{r}3 . \\
1 \\
2\end{array}$ & $\begin{array}{r}1.4 \\
45\end{array}$ \\
\hline $\begin{array}{l}\text { 5.Cleaning workers } \\
\text { put a warning sign } \\
\text { when the start } \\
\text { sanitizing work } \\
\text { floors }\end{array}$ & $\begin{array}{l}5 \\
5\end{array}$ & $\begin{array}{l}1 \\
2 \\
5\end{array}$ & $\begin{array}{l}1 \\
2 \\
1\end{array}$ & $\begin{array}{r}27 \\
.4\end{array}$ & 89 & $\begin{array}{c}2 \\
0 . \\
2\end{array}$ & $\begin{array}{l}9 \\
5\end{array}$ & $\begin{array}{c}2 \\
1 . \\
5\end{array}$ & $\begin{array}{l}8 \\
1\end{array}$ & $\begin{array}{c}1 \\
8 . \\
4\end{array}$ & $\begin{array}{r}2 . \\
9 \\
4\end{array}$ & $\begin{array}{r}1.3 \\
13\end{array}$ \\
\hline $\begin{array}{l}\text { 6.Protective } \\
\text { barriers are } \\
\text { available to be } \\
\text { placed on doors and } \\
\text { windows to prevent } \\
\text { direct contact with } \\
\text { patients }\end{array}$ & $\begin{array}{l}7 \\
8\end{array}$ & $\begin{array}{l}1 \\
7 \\
.\end{array}$ & $\begin{array}{l}9 \\
6\end{array}$ & $\begin{array}{r}21 \\
.8\end{array}$ & 78 & $\begin{array}{c}1 \\
7 . \\
7\end{array}$ & $\begin{array}{l}9 \\
6\end{array}$ & $\begin{array}{c}2 \\
1 . \\
8\end{array}$ & $\begin{array}{l}9 \\
3\end{array}$ & $\begin{array}{c}2 \\
1 . \\
1\end{array}$ & $\begin{array}{r}2 . \\
9 \\
3\end{array}$ & $\begin{array}{r}1.4 \\
09\end{array}$ \\
\hline $\begin{array}{l}\text { 7.There are } \\
\text { indications on the } \\
\text { floors for social } \\
\text { distancing of } 2 \\
\text { meters or one and a } \\
\text { half meters }\end{array}$ & $\begin{array}{l}5 \\
1\end{array}$ & $\begin{array}{l}1 \\
1 \\
\dot{6}\end{array}$ & $\begin{array}{l}6 \\
9\end{array}$ & $\begin{array}{r}15 \\
.6\end{array}$ & 90 & $\begin{array}{c}2 \\
0 . \\
4\end{array}$ & $\begin{array}{l}1 \\
1 \\
2\end{array}$ & $\begin{array}{c}2 \\
5 . \\
4\end{array}$ & $\begin{array}{l}1 \\
1 \\
9\end{array}$ & $\begin{array}{c}2 \\
7 . \\
0\end{array}$ & $\begin{array}{r}2 . \\
5 \\
9\end{array}$ & $\begin{array}{r}1.3 \\
39\end{array}$ \\
\hline $\begin{array}{l}\text { 8.Wash } \\
\text { basins(sink)are } \\
\text { available at the } \\
\text { exits of the rooms }\end{array}$ & $\begin{array}{l}6 \\
3\end{array}$ & $\begin{array}{l}1 \\
4 \\
3 \\
\end{array}$ & $\begin{array}{l}1 \\
3 \\
3\end{array}$ & $\begin{array}{r}30 \\
.2\end{array}$ & 98 & $\begin{array}{c}2 \\
2 . \\
2\end{array}$ & $\begin{array}{l}8 \\
4\end{array}$ & $\begin{array}{c}1 \\
9 . \\
0\end{array}$ & $\begin{array}{l}6 \\
3\end{array}$ & $\begin{array}{r}1 \\
4 . \\
3\end{array}$ & $\begin{array}{c}3 . \\
1 \\
1\end{array}$ & $\begin{array}{r}1.2 \\
75\end{array}$ \\
\hline $\begin{array}{l}\text { 9.The work } \\
\text { environment is } \\
\text { suitable for } \\
\text { personal protective } \\
\text { equipment to } \\
\text { wearing } \\
\text { throughout the } \\
\text { working hours }\end{array}$ & $\begin{array}{l}8 \\
5\end{array}$ & $\begin{array}{l}1 \\
9 \\
3\end{array}$ & $\begin{array}{l}1 \\
7 \\
9\end{array}$ & $\begin{array}{r}40 \\
.6\end{array}$ & 93 & $\begin{array}{c}2 \\
1 . \\
1\end{array}$ & $\begin{array}{l}4 \\
4\end{array}$ & $\begin{array}{c}1 \\
0 . \\
0\end{array}$ & $\begin{array}{l}4 \\
0\end{array}$ & $\begin{array}{r}9 . \\
1\end{array}$ & $\begin{array}{r}3 . \\
5 \\
1\end{array}$ & $\begin{array}{l}1.1 \\
76\end{array}$ \\
\hline
\end{tabular}




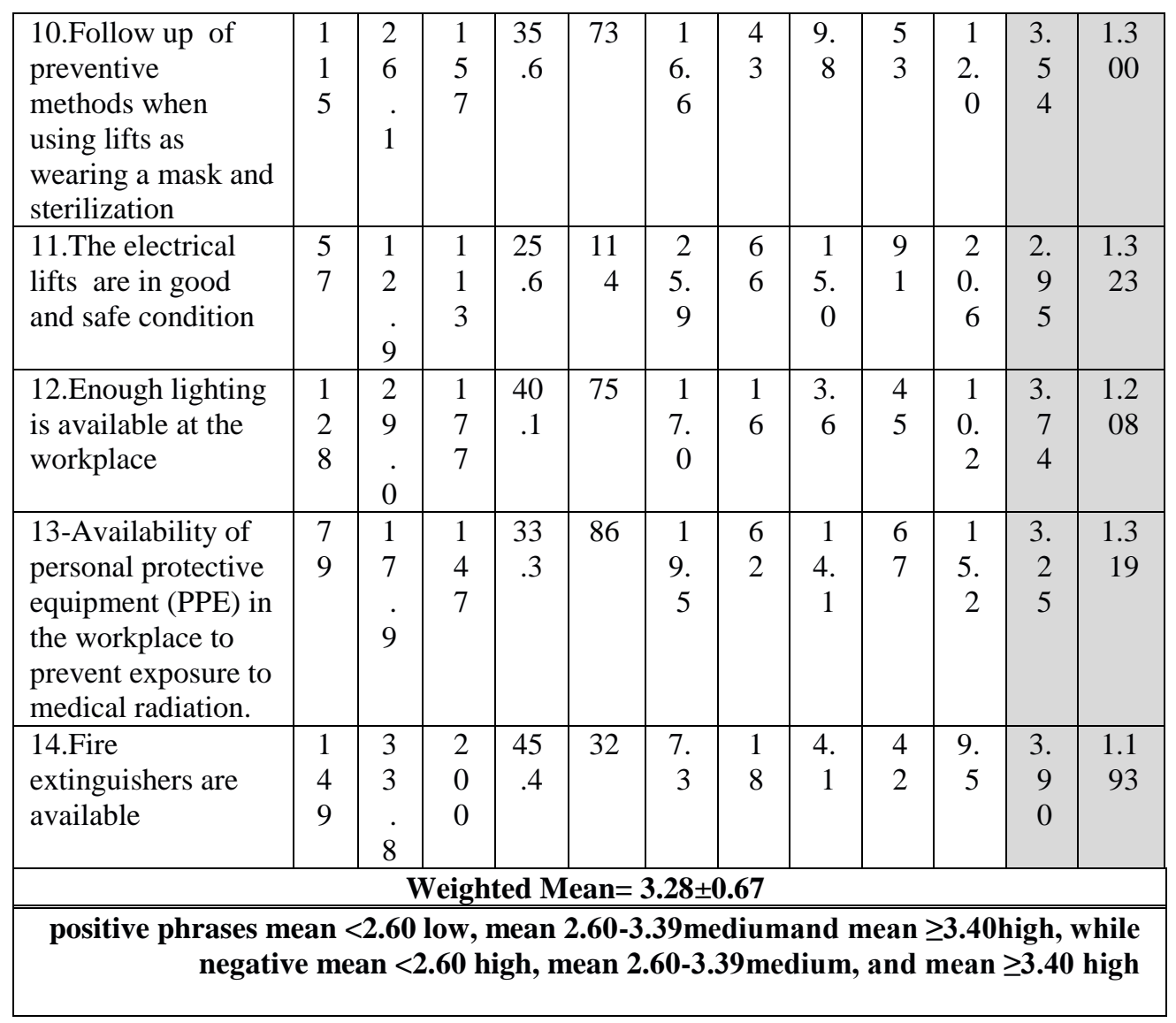

Table 2.1, the result of the found that study the highest percentage 71(74.7\%) of the study population had a good score (low risk) about physical hazards in Al- Zahraa teaching hospital followed by $58.3 \%$ in Al-Shaheed Fairooz hospital. While the overall percentage of physical hazards in the studied hospitals were reached 245 (55.6\%) (Low risk) and high risk reached 196(44.4\%) The differences between the four hospitals concerning the scoring level were statistically significant $\mathrm{P}$ value equal 0.05 .

Table 2.1. The Relationship between the Study Hospitals and Total Risk Score of The Physical Hazards.

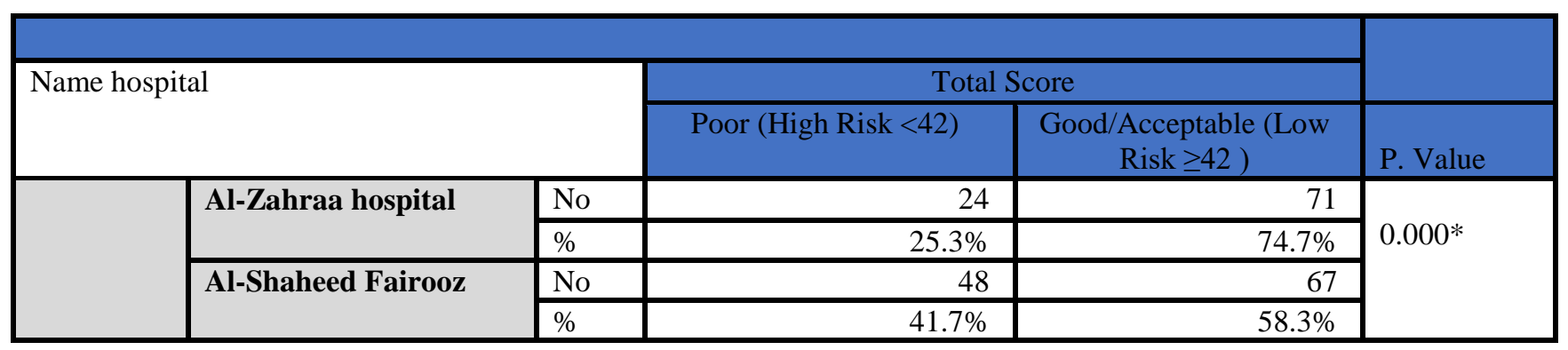




\begin{tabular}{|l|l|l|r|r|}
\hline \multirow{4}{*}{ Al-swiara } & No & 75 & 54 \\
\cline { 3 - 5 } & & $\%$ & $58.1 \%$ & $41.9 \%$ \\
\cline { 3 - 5 } & \multirow{2}{*}{ al-Nuamania } & No & 49 & 53 \\
\cline { 3 - 5 } & & $\%$ & $48.0 \%$ & $52.0 \%$ \\
\hline \multirow{2}{*}{ Total } & No & 196 & 245 \\
\cline { 3 - 5 } & & $\%$ & $44.4 \%$ & $55.6 \%$ \\
\hline
\end{tabular}

Table 2.2 represents the relationship between the demographic characteristics of the studied sample concerning all questions was not statistically significant $\mathrm{P}>0.05$, except social status $\mathrm{P}$ value equal 0.047 , professional $\mathrm{P}$ value equal 0.025 , and experience years $\mathrm{P}$ value equal 0.026 were significant.

Table 2.2. The Relationship between Demographic Characteristics of Healthcare Workers and The Total

\begin{tabular}{||l||l|l||c||}
\hline \hline Association & Total Physical Hazards Score \\
\hline Socio-demographic & DF & Chi-Square $\left(\boldsymbol{X}^{\mathbf{2}}\right)$ & P. value \\
\hline \hline age groups & 1 & 0.314 & 0.575 \\
\hline \hline Gender & 1 & 0.440 & 0.507 \\
\hline \hline Residence & 1 & 0.934 & 0.334 \\
\hline \hline Educational level & 3 & 6.079 & 0.108 \\
\hline \hline social status & 2 & 6.129 & $0.047^{*}$ \\
\hline \hline Professional & 6 & 14.480 & $0.025^{*}$ \\
\hline \hline experience years & 1 & 4.962 & $0.026^{*}$ \\
\hline \hline Place of working & 10 & 17.329 & 0.067 \\
\hline \hline
\end{tabular}

Table 3 Represent the distribution of HCWs according to chemical hazards. The results of this study indicated that the mean regarding (labels are placed to explain which detergents to avoided mix with other chemicals it may cause toxic gas) was (3.73) were rest on agreed respond level. While the SD of (1.462).

Table 3. The Distribution of Healthcare Workers According to chemical Hazards.

\begin{tabular}{|c|c|c|c|c|c|c|c|c|c|c|c|c|}
\hline \multirow[t]{2}{*}{ Chemical hazard } & \multicolumn{2}{|c|}{$\begin{array}{l}\text { Strongly } \\
\text { agree }\end{array}$} & \multicolumn{2}{|c|}{ Agree } & \multicolumn{2}{|c|}{ Neutral } & \multicolumn{2}{|c|}{ Disagree } & \multicolumn{2}{|c|}{$\begin{array}{l}\text { Strongly } \\
\text { Disagree }\end{array}$} & \multirow[t]{2}{*}{$\begin{array}{l}\mathrm{Me} \\
\text { an }\end{array}$} & \multirow[t]{2}{*}{ SD } \\
\hline & No & $\%$ & No & $\%$ & $\begin{array}{l}\mathrm{N} \\
\mathrm{O}\end{array}$ & $\%$ & No & $\%$ & No & $\%$ & & \\
\hline $\begin{array}{l}\text { 1. Sterilizers and } \\
\text { disinfects are } \\
\text { used }\end{array}$ & $\begin{array}{r}12 \\
0\end{array}$ & $\begin{array}{r}27 \\
.2\end{array}$ & $\begin{array}{r}11 \\
6\end{array}$ & $\begin{array}{r}26 \\
.3\end{array}$ & $\begin{array}{l}4 \\
4\end{array}$ & $\begin{array}{r}10 \\
.0\end{array}$ & 80 & $\begin{array}{r}18 \\
.1\end{array}$ & 81 & $\begin{array}{r}18 \\
.4\end{array}$ & $\begin{array}{r}3 . \\
26\end{array}$ & $\begin{array}{r}1.4 \\
85\end{array}$ \\
\hline $\begin{array}{l}\text { 2.Guidance } \\
\text { labels are placed }\end{array}$ & 55 & $\begin{array}{r}12 \\
.5\end{array}$ & 87 & $\begin{array}{r}19 \\
.7\end{array}$ & $\begin{array}{l}8 \\
9\end{array}$ & $\begin{array}{r}20 \\
.2\end{array}$ & $\begin{array}{r}11 \\
6\end{array}$ & $\begin{array}{r}26 \\
.3\end{array}$ & 94 & $\begin{array}{r}21 \\
.3\end{array}$ & $\begin{array}{l}2 . \\
76\end{array}$ & $\begin{array}{r}1.3 \\
25\end{array}$ \\
\hline
\end{tabular}




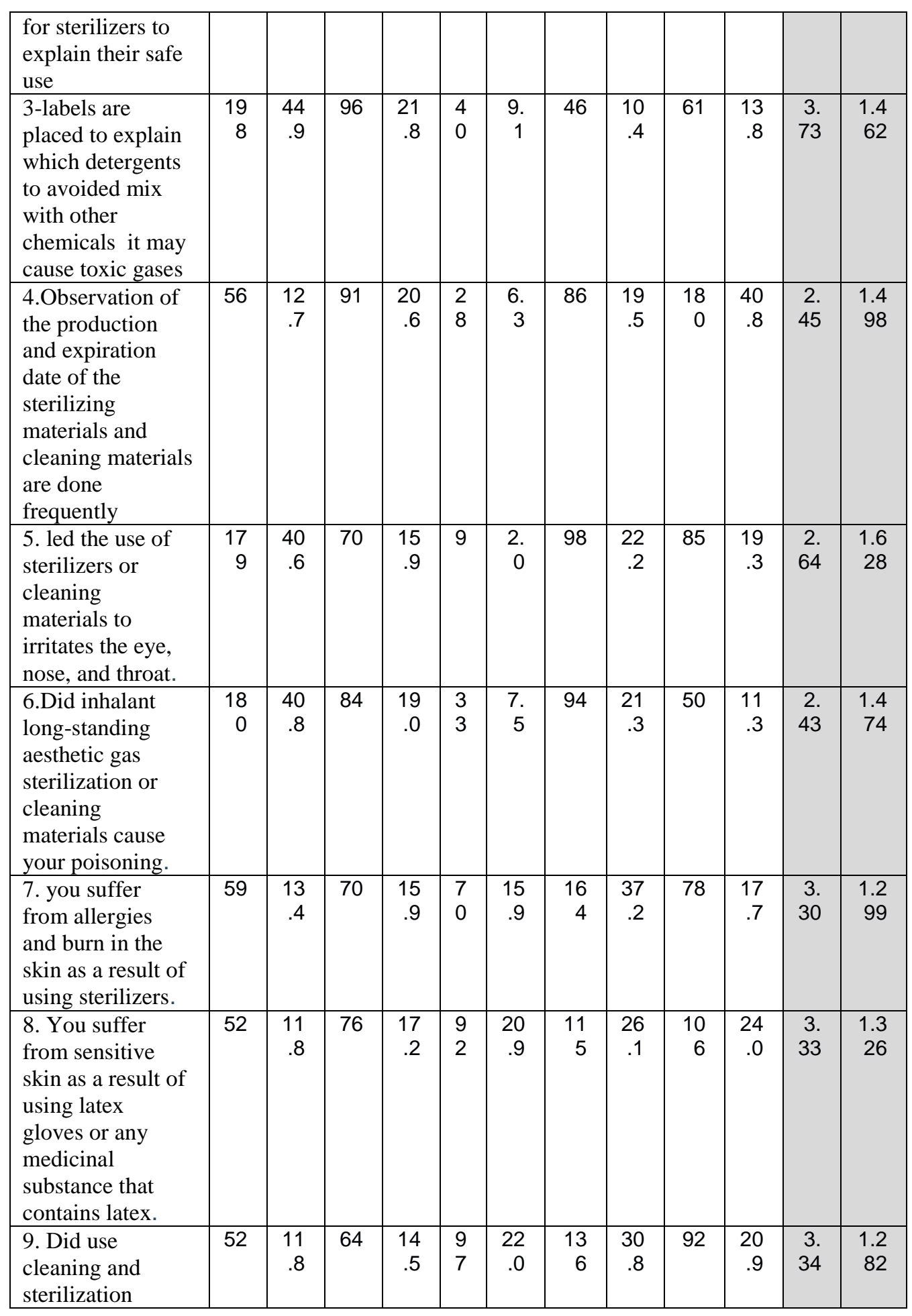




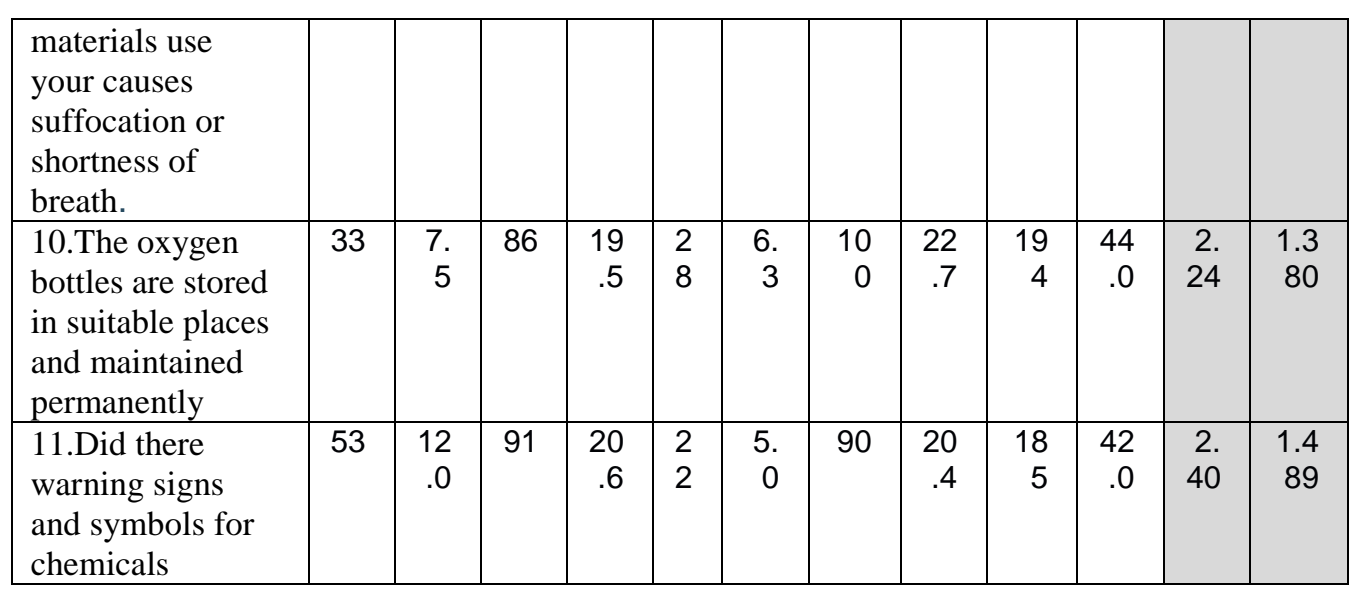

Table 3.1 Represent the total score percentage of chemical hazards level was found poor score $241(54.6 \%)$ and high score $200(45.4 \%)$ in the study. The association between the four hospitals concerning the scoring level was statistically significant $\mathrm{P}$ value equal to 0.047 .

Table 3.1. The Relationship between the Study Hospitals and Total Risk Score of the Chemical Hazards.

\begin{tabular}{|c|c|c|c|c|c|}
\hline & & & & & P. Value \\
\hline \multicolumn{3}{|c|}{ Name hospital } & \multicolumn{2}{|c|}{ Total Score } & \\
\hline & & & \multirow{2}{*}{$\begin{array}{r}\text { Poor (High Risk } \\
<33) \\
64\end{array}$} & \multirow{2}{*}{$\begin{array}{r}\text { Good/Acceptable } \\
(\text { Low Risk } \geq 33) \\
31\end{array}$} & \multirow{11}{*}{$0.047^{*}$} \\
\hline & Al-Zahraa & No & & & \\
\hline & nospital & $\%$ & $67.4 \%$ & $32.6 \%$ & \\
\hline & Al-Shaheed & No & 58 & 57 & \\
\hline & & $\%$ & $50.4 \%$ & $49.6 \%$ & \\
\hline & Al-swiara & No & 67 & 62 & \\
\hline & & $\%$ & $51.9 \%$ & $48.1 \%$ & \\
\hline & al-Nuamania & No & 52 & 50 & \\
\hline & & $\%$ & $51.0 \%$ & $49.0 \%$ & \\
\hline \multirow{2}{*}{\multicolumn{2}{|c|}{ Total }} & No & 241 & 200 & \\
\hline & & $\%$ & $54.6 \%$ & $45.4 \%$ & \\
\hline
\end{tabular}


Table 3.2 Represent the Relationship between the demographic characteristics of the studied sample concerning all questions was not statistically significant $\mathrm{P}>0.05$, as appear in Table 7 . Except gender was significant P-value equal 0.049.

Table 3.2. The Relationship between Demographic Characteristics of healthcare workers and the Total risk Score about Chemical hazards.

\begin{tabular}{|c||c|c|c||}
\hline \multicolumn{1}{|c||}{ Association } & \multicolumn{3}{|c|}{ Total Chemical Hazards Score } \\
\hline \hline Socio-demographic & DF & Chi-Square $\left(\boldsymbol{X}^{\mathbf{2}}\right)$ & P. value \\
\hline \hline age groups & 1 & 0.866 & 0.352 \\
\hline \hline Gender & 1 & 3.882 & $0.049^{*}$ \\
\hline \hline Residence & 1 & 0.045 & 0.832 \\
\hline \hline Educational level & 3 & 5.289 & 0.152 \\
\hline \hline Social status & 2 & 2.213 & 0.331 \\
\hline \hline Professional & 6 & 2.717 & 0.843 \\
\hline Place of working & 10 & 0.495 & 0.482 \\
\hline \hline
\end{tabular}

Table 4 Represent the distribution of HCW according to prevention and control methods in health institutions. The mean for question (1)( training courses are conducted to educate health care workers about coronavirus )was(2.24), for question( 21) (the first aid kit is available and usable ) was(3.74), were resting on agree to responses level. While SD for question (1) was(1.380), for question (21)was(1.060).

Table 4. Distribution of Healthcare Workers according to the Prevention and Control Methods.

\begin{tabular}{|c|c|c|c|c|c|c|c|c|c|c|c|c|}
\hline Prevention and Methods & \multicolumn{2}{|c|}{ Strongly agree } & \multicolumn{2}{|l|}{ Agree } & \multicolumn{2}{|c|}{ Neutral } & \multicolumn{2}{|c|}{ Disagree } & \multicolumn{2}{|c|}{$\begin{array}{l}\text { Strongly } \\
\text { Disagree }\end{array}$} & M & SD \\
\hline $\begin{array}{l}\text { 1.Training courses are } \\
\text { conducted to educate } \\
\text { health care workers } \\
\text { about corona virus }\end{array}$ & 33 & 7.5 & 86 & 19.5 & 28 & 6.3 & 100 & 22.7 & 194 & 44.0 & 2.24 & 1.380 \\
\hline $\begin{array}{l}\text { 2.instruction on } \\
\text { infection prevention and } \\
\text { control is available on } \\
\text { coronavirus }\end{array}$ & 53 & 12.0 & 91 & 20.6 & 22 & 5.0 & 90 & 20.4 & 185 & 42.0 & 2.40 & 1.489 \\
\hline $\begin{array}{l}\text { 3.Discouraging a direct } \\
\text { contact with } \\
\text { employees(hugging and } \\
\text { shaking hand) }\end{array}$ & 61 & 13.8 & 88 & 20.0 & 27 & 6.1 & 83 & 18.8 & 182 & 41.3 & 2.46 & 1.519 \\
\hline
\end{tabular}




\begin{tabular}{|c|c|c|c|c|c|c|c|c|c|c|c|c|}
\hline $\begin{array}{l}\text { 4.the workplace shall be } \\
\text { disinfected at } \\
\text { appropriate intervals }\end{array}$ & 56 & 12.7 & 91 & 20.6 & 28 & 6.3 & 86 & 19.5 & 180 & 40.8 & 2.45 & 1.498 \\
\hline $\begin{array}{l}\text { 5. the medical devices } \\
\text { are disinfected before } \\
\text { and after use }\end{array}$ & 85 & 19.3 & 98 & 22.2 & 9 & 2.0 & 70 & 15.9 & 179 & 40.6 & 2.64 & 1.628 \\
\hline $\begin{array}{l}\text { 6.soap and tissue paper } \\
\text { are provided e near the } \\
\text { handwashing basin }\end{array}$ & 50 & 11.3 & 94 & 21.3 & 33 & 7.5 & 84 & 19.0 & 180 & 40.8 & 2.43 & 1.474 \\
\hline $\begin{array}{l}\text { 7.hands washing before } \\
\text { entering the work } \\
\text { environment and after } \\
\text { takeoff the personal } \\
\text { protective equipment } \\
\text { and when leaving the } \\
\text { work environment }\end{array}$ & 58 & 13.2 & 96 & 21.8 & 9 & 2.0 & 85 & 19.3 & 193 & 43.8 & 2.41 & 1.532 \\
\hline $\begin{array}{l}\text { 8.Avoidance frequent } \\
\text { contact with common } \\
\text { surfaces where possible } \\
\text { (leaving doors open } \\
\text { where possible) }\end{array}$ & 111 & 25.2 & 231 & 52.4 & 71 & 16.1 & 22 & 5.0 & 6 & 1.4 & 3.95 & 1.857 \\
\hline $\begin{array}{l}\text { 9.prevent the } \\
\text { gatherings in elevators } \\
\text { or elevators for } \\
\text { transporting people } \\
\text { which is considered } \\
\text { unsafe under the } \\
\text { current conditions of } \\
\text { the coronavirus and } \\
\text { beware of contact } \\
\text { points in elevators }\end{array}$ & 85 & 19.3 & 199 & 45.1 & 93 & 21.1 & 42 & 9.5 & 22 & 5.0 & 3.64 & 1.052 \\
\hline $\begin{array}{l}\text { 10. the employees and } \\
\text { auditors who enter the } \\
\text { health institution are } \\
\text { examined searching for } \\
\text { symptoms of } \\
\text { coronavirus }\end{array}$ & 33 & 7.5 & 86 & 19.5 & 28 & 6.3 & 100 & 22.7 & 194 & 44.0 & 2.24 & 1.380 \\
\hline $\begin{array}{l}\text { 11.Availability of } \\
\text { adequate quantities of } \\
\text { personal protective } \\
\text { equipment(mask, } \\
\text { gloves, eyeglasses, } \\
\text { boots, work suit) }\end{array}$ & 53 & 12.0 & 91 & 20.6 & 22 & 5.0 & 90 & 20.4 & 185 & 42.0 & 2.40 & 1.489 \\
\hline $\begin{array}{l}\text { 12.Provide personal } \\
\text { protective equipment in } \\
\text { appropriate sizes for }\end{array}$ & 61 & 13.8 & 88 & 20.0 & 27 & 6.1 & 83 & 18.8 & 182 & 41.3 & 2.46 & 1.519 \\
\hline
\end{tabular}




\begin{tabular}{|c|c|c|c|c|c|c|c|c|c|c|c|c|}
\hline $\begin{array}{l}\text { every employee or } \\
\text { person visiting the } \\
\text { workplace }\end{array}$ & & & & & & & & & & & & \\
\hline $\begin{array}{l}\text { 13.Prevention } \\
\text { equipment is examined } \\
\text { periodically and } \\
\text { maintained and } \\
\text { replaced when needed }\end{array}$ & 56 & 12.7 & 91 & 20.6 & 28 & 6.3 & 86 & 19.5 & 180 & 40.8 & 2.45 & 1.498 \\
\hline $\begin{array}{l}\text { 14.Personal prevention } \\
\text { equipment is eliminated } \\
\text { in safe ways do not } \\
\text { pollute the work } \\
\text { environment and others }\end{array}$ & 85 & 19.3 & 98 & 22.2 & 9 & 2.0 & 70 & 15.9 & 179 & 40.6 & 2.64 & 1.628 \\
\hline $\begin{array}{l}\text { 15.the wounds are } \\
\text { covered before the } \\
\text { laboratory enter }\end{array}$ & 50 & 11.3 & 94 & 21.3 & 33 & 7.5 & 84 & 19.0 & 180 & 40.8 & 2.43 & 1.474 \\
\hline $\begin{array}{l}\text { 16. the jewelry is } \\
\text { covered(it should not } \\
\text { affect the gloves)and } \\
\text { removed before } \\
\text { entering any workplace } \\
\text { where this is required }\end{array}$ & 58 & 13.2 & 96 & 21.8 & 9 & 2.0 & 85 & 19.3 & 193 & 43.8 & 2.41 & 1.532 \\
\hline $\begin{array}{l}\text { 17.portable electronic } \\
\text { devices are kept in } \\
\text { areas that cannot be } \\
\text { contaminated and } \\
\text { disinfected frequently }\end{array}$ & 103 & 23.4 & 220 & 49.9 & 69 & 15.6 & 33 & 7.5 & 16 & 3.6 & 3.82 & 0.993 \\
\hline $\begin{array}{l}\text { 18. A warning signs } \\
\text { exist in biological } \\
\text { laboratories have }\end{array}$ & 101 & 22.9 & 178 & 40.4 & 95 & 21.5 & 39 & 8.8 & 28 & 6.3 & 3.65 & 1.117 \\
\hline $\begin{array}{l}\text { 19. Records of waste } \\
\text { removal destruction } \\
\text { and treatment are } \\
\text { available }\end{array}$ & 84 & 19.0 & 183 & 41.5 & $\begin{array}{r}10 \\
0\end{array}$ & 22.7 & 40 & 9.1 & 34 & 7.7 & 3.55 & 1.129 \\
\hline $\begin{array}{l}\text { 20.waste workers are } \\
\text { being drawn to } \\
\text { coronavirus-related } \\
\text { waste }\end{array}$ & 110 & 24.9 & 190 & 43.1 & 77 & 17.5 & 36 & 8.2 & 28 & 6.3 & 3.72 & 1.117 \\
\hline $\begin{array}{l}21 . f i r s t \text { aid kit is } \\
\text { available and usable }\end{array}$ & 99 & 22.4 & 209 & 47.4 & 79 & 17.9 & 28 & 6.3 & 26 & 5.9 & 3.74 & 1.060 \\
\hline $\begin{array}{l}\text { 22.A published } \\
\text { contingency plan is } \\
\text { available }\end{array}$ & 33 & 7.5 & 86 & 19.5 & 28 & 6.3 & 100 & 22.7 & 194 & 44.0 & 2.24 & 1.380 \\
\hline
\end{tabular}




\begin{tabular}{|l|l|l|l|l|l|l|l|l|l|l|l|l|}
\hline $\begin{array}{l}\text { 23.A workplace } \\
\text { response plan is an } \\
\text { available case someone } \\
\text { develops symptoms of } \\
\text { coronavirus and } \\
\text { employees are aware of } \\
\text { them }\end{array}$ & 53 & 12.0 & 91 & 20.6 & 22 & 5.0 & 90 & 20.4 & 185 & 42.0 & 2.40 & 1.489 \\
\hline & & & & & & & & & & & &
\end{tabular}

Fig 1 Represent the total score prevention and control methods. The highest percentage of the studied sample which reached 266(60.0\%) had a poor score (high risk) regarding prevention and control methods, while reached to $175(40.0 \% \%)$ of them had a good score (low risk) toward it.

\section{Prevention and Control methods Score}

— Low risk (175) \# High risk (266)

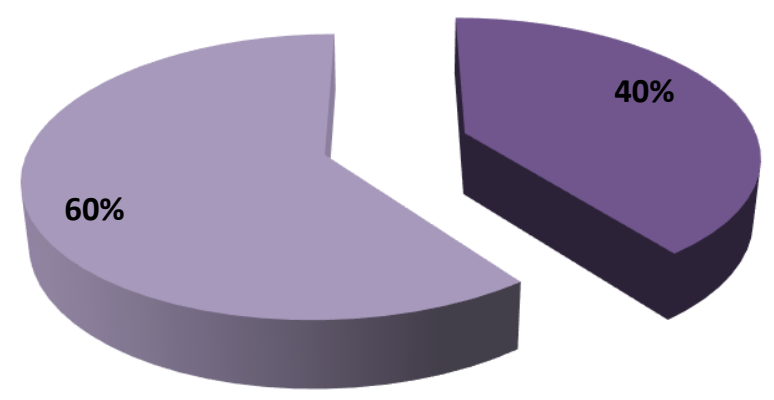

Fig 1. The total score of the Prevention and Control Methods.

Table 5 represents Pearson's correlation coefficients physical hazards, and prevention and control methods. It shows that there is a highly significant positive correlation (P-value $<0.01$ ) between physical hazards and prevention and control methods $\left(\mathrm{r}=0.144^{*}\right)$.

Table 5. Pearson's correlation coefficients physical hazards, and prevention and control methods.

\begin{tabular}{||l|l|c|}
\hline \multicolumn{2}{|l|}{} & physical hazard \\
\hline \hline \multirow{2}{*}{$\begin{array}{l}\text { Prevention and } \\
\text { control methods }\end{array}$} & $\begin{array}{l}\text { Pearson } \\
\text { Correlation }\end{array}$ & $0.144^{* *}$ \\
\cline { 2 - 3 } & Sig. (2-tailed) & 0.002 \\
\hline
\end{tabular}




\begin{tabular}{|l|l|}
\hline $\mathrm{N}$ & 441 \\
\hline
\end{tabular}

Table 6 represents Pearson's correlation coefficients chemical hazards, and prevention and control methods. It shows that there is a highly significant positive correlation $(\mathrm{P}$-value $<0.01)$ between chemical hazards and prevention and control methods $\left(\mathrm{r}=0.770^{*}\right)$.

Table 6. Pearson's correlation coefficients chemical hazards, and prevention and control methods.

\begin{tabular}{|c|c|c|}
\hline & & Chemical hazards \\
\hline \multirow{4}{*}{$\begin{array}{c}\text { Prevention and } \\
\text { control } \\
\text { methods }\end{array}$} & Pearson & $0.770^{* *}$ \\
\hline & Correlation & \\
\hline & Sig. (2-tailed) & 0.000 \\
\hline & $\mathrm{N}$ & 441 \\
\hline
\end{tabular}

\section{Discussion}

The present study found that 254(57.6\%) were in the age group of health care workers $<30$ years. These results are consistent with the previous study was done in Palestine hospitals [7], which found that $60 \%$ of the study participants fall included the age group (20-30 years) This may be due to the increasing number of graduates from medical institutes and colleges in Iraq and Their enrolment in the direct job more than before. in this study, there was a distinct female's preponderance $242(54.9 \%)$. These results agreed with the study findings done in Nigeria [6], which found that most of the participants were females, and another similar study in India [9] revealed that the highest percentage (80\%) of the study samples were females. Furthermore, these results are consistent with the study findings done in Riyadh, Saudi Arabia [10], which found the participants proportion in the study for females higher than males. But, these results differ from the findings study in Palestine [11] who found that most participants were males. The results of this study indicated that $265(60.1 \%)$ of the study population were married, and $161(36.5 \%)$ of Participants were single in studied hospitals. These results are consistent with the study done in Nigeria[8], which revealed that the highest percentage $(63.0 \%)$ of the study samples were married. The current findings that the first rank of health care workers were nurses $125(28.3 \%)$ followed which reached to $107(24.3 \%)$ of medical assistants, laboratory assistants, and radiology assistants in all studied hospitals. While the overall frequency of Technicians was $86(19.5 \%)$ in all hospitals. This may be due to the increasing number of private and government colleges that graduate large number of nurses .These results agreed with the study conducted in the Northern West Bank Hospital Palestine [12], which found that the first rank of health care workers were practical nurses $(46.4 \%)$.

In this study, most of the participants respond with "agree and strongly agree" regarding" the electrical lifts are in a good and safe condition" and" enough lighting is available at the workplace". These findings agreed with the previous study in Egypt[13]. Which found that most of HCWs reported that electrical installations and lighting were in good condition

This study revealed there are differences between the four hospitals concerning the physical hazards level were statistically significant $P$ value equal 0.05 . These findings were agreed to the study conducted in a Greek hospital [14], which found that most participants declared low to 
medium levels of risk concerning physical hazards. But, these results disagreed with the study conduct in Egypt[15], which found that physical hazards were the first rank among radiation health teams compared to other occupational hazards.

The association between the four hospitals concerning the chemical hazard scoring level was statistically significant $\mathrm{P}$ value equal to 0.047 . These results disagree with another study conducted in Benin-city, Nigeria [16], which found that chemical hazards levels were $68 \%$ among health care workers and the association between the study hospitals concerning the exposure level were statistically significant $P$ value equal 0.005 .

The study was done in Palestine, Almurr, 2013who reported that respondents do not take training regarding safety practices [12]. Also, a study, conducted in a European Gaza hospital Saqer, 2014) found there is a lack of occupational training for health care providers towards knowledge of occupational health and safety risks at European Gaza Hospital. The finding agreed with our result in this study [17].

In Palestine, a study revealed that only $(27.3 \%)$ of health care personnel indicated the lack of infection-prevention equipment such as gloves, hats, closed shoes, and work suits. This result disagreed with the study of our findings in Palestine [18]. A study conduct in Gaza [19], who showed apparent progress in adherence of healthcare workers in the implementation of infection prevention. This finding disagrees with our results in this study.

\section{Conclusion}

There are some following conclusions:

1- The study showed that the hazards in hospitals were moderate for chemical hazards followed by physical hazards.

2- The study revealed that the less commitment to prevention and control methods, the greater the risks to workers.

3- The study revealed that the relationship between the study sample and the physical hazards with the regarded to demographic characteristics are (social status, professional and experience years) $\mathrm{p}>0.05$.

\section{References}

[1] Younis, NM., Hussein, AA., Ahmed, MM., \& Younis, NM. Quality of life and occupational hazards among cement factory workers in Mosul city. QoL and Occupational Hazards among Cement Factory Workers.2021, 24(2), 1-8.

[2] Abdel-Wahed, A., Alazab, RM., Elsaidy, W H., Imam, M E.-H., \& Ghandour, A A. Risk assessment of physical health hazards in Al-Azhar University Hospital in new Damietta, Egypt. The Egyptian Journal of Hospital Medicine.2013 , 53(1), 1019-1035.

[3] Khamtuikrua C, Suksompong S. Awareness about radiation hazards and knowledge about radiation protection among healthcare personnel: A quaternary care academic center-based study. SAGE open Med. 2020;8:1-8.

[4] Erkan I, Yarenoglu A, Yukseloglu EH, Ulutin HC. The investigation of radiation safety awareness among healthcare workers in an education and research hospital. Int J Radiat Res. 2019;17(3):447-53.

[5] Bhusnure OG, Dongare RB, Gholve SB, Giram PS. Chemical hazards and safety management in pharmaceutical industry. J Pharm Res. 2018;12(3):357-69.

[6] United State Food and Drug Administration. Personal Protective Equipment for Infection Control [Internet]. 2020 [cited 2021 Apr 14]. Available from: https://www.fda.gov/medical-devices/generalhospital-devices-and-supplies/personal-protective-equipment-infection-control 
[7] Al-Khatib IA, El Ansari W, Areqat TA, Darkhawaja RA, Mansour SH, Tucktuck MA, et al. Occupational safety precautions among nurses at four hospitals, Nablus district, Palestine. Int J Occup Environ Med. 2015;6(4):243-6.

[8] Seidat Moyosore Ogunnaike MOA. Occupational hazard preventive measures among nurses in a Nigerian tertiary health institution. Nurs Care Open Access J [Internet]. 2020;7(1):20-5. Available from: https://medcraveonline.com/NCOAJ/NCOAJ-07-00212.pdf

[9] Chelladurai UM, Suga SSD, Myneni S, Sivagananam P, Ravikumar D, Kumar MR, et al. KNOWLEDGE, ATTITUDES ON OCCUPATIONAL HEALTH HAZARDS AND SAFETY (OHHS) AMONG THE HEALTHCARE WORKERS IN A TERTIARY CARE HOSPITAL. J Crit Rev. 2020;7(14):1540-8.

[10] Alshalani AJ, Salama KF. Assessment of Occupational Safety Practices Among Medical Laboratory Staff in Governmental Hospitals in Riyadh, Saudi Arabia. J Saf Stud. 2019;5(1):1-23.

[11] Alqam TIS. Occupational hazards among laboratory workers in Palestinian governmental hospitals in the West Bank.Al- Quds University [Internet]. Master Thesis; 2013. Available from: https://core.ac.uk/download/pdf/287328913.pdf

[12] Almurr BJ. Knowledge and Practice of Standard Precaution and Sharp Injures among Nurses in the Northern West Bank Hospitals; Palestine [Internet]. 2013. Available from: https://repository.najah.edu/bitstream/handle/20.500.11888/7825/Bushra

AlMurr_0.pdf?sequence=1\&isAllowed=y

[13] Abdel-Wahed A, Alazab RM, Elsaidy WH, Imam ME-H, Ghandour AA. Risk assessment of physical health hazards in Al-Azhar University Hospital in new Damietta, Egypt. Egypt $\mathrm{J}$ Hosp Med. 2013;53(1):1019-35.

[14] Tziaferi SG, Sourtzi P, Kalokairinou A, Sgourou E, Koumoulas E, Velonakis E. Risk assessment of physical hazards in greek hospitals combining staff's perception, experts' evaluation and objective measurements. Saf Health Work. 2011;2(3):260-72.

[15] Eliwa SM, Sorour AS, Mahmoud SF. Occupational Health Hazards and Protective Measures among Radiation Health Team. Zagazig Nurs J. 2018;14(2):48-63.

[16] Omoijiade EN. An assessment of laundry workers exposure to workplace hazards in secondary and tertiary health facilities in Benin-city, Nigeria. MOJ public Heal. 2018;7(5):252-9.

[17] Saqer AM. Assessment of Health and Safety Risk among Health Care Providers in European Gaza Hospital. (Thesis) Islamic University-Gaza,Faculty of Science - Environmental Health. 2014.

[18] El Attar SM. Assessment of health and safety risk among health care providers at Al Shifa Medical Complex [Internet].2016. Available from: https://iugspace.iugaza.edu.ps/bitstream/handle/20.500.12358/21320/file_1.pdf?sequence=1\&isAllowed $=\mathrm{y}$

[19] Aldirawi A, Aljerjawy M, abu Mustafa A. Adherence to infection prevention and control protocol in neonatal intensive care units in governmental hospitals in Gaza Strip-A comparative study. Int J Recent Acad Res. 2019;1(7):300-4. 